\title{
Pairing of 1-hexyl-3-methylimidazolium and tetrafluoroborate ions in $n$-pentanol
}

\author{
P. Zhu, 1 (a) L. R. Pratt, 1, b) and K. D. Papadopoulos ${ }^{1,(c)}$ \\ Department of Chemical and Biomolecular Engineering, Tulane University, New Orleans, \\ LA 70118
}

(Dated: 1 November 2018)

Molecular dynamics simulations are obtained and analyzed to study pairing of 1-hexyl-3-methylimidazolium and tetrafluoroborate ions in $n$-pentanol, in particular by evaluating the potential-of-mean-force between counter ions. The present molecular model and simulation accurately predicts the dissociation constant $K_{\mathrm{d}}$ in comparison to experiment, and thus the behavior and magnitudes for the ion-pair pmf at molecular distances, even though the dielectric constant of the simulated solvent differs from the experimental value by about $30 \%$. A naive dielectric model does not capture molecule structural effects such as multiple conformations and binding geometries of the $\mathrm{Hmim}^{+}$and $\mathrm{BF}_{4}{ }^{-}$ion-pairs. Mobilities identify multiple time-scale effects in the autocorrelation of the random forces on the ions, and specifically a slow, exponential time-decay of those long-ranged forces associated here with dielectric friction effects.

\section{INTRODUCTION}

Ion-pair encounter, binding, and dissociation is central to solution chemistry, including chemistry in organic solvents, 1 and to understanding specific electrolyte solutions in a wide range of practical settings. ${ }^{2}$ We recently obtained an experimental determination of ion-pairing specifically of 1-hexyl-3-methylimidazolium tetrafluoroborate in $n$-pentanol $[3$ Those results encouraged us to pursue detailed testing of the molecular-scale description of ion pairing and dynamics for that system. We report results of that testing here. We report, among other results, evaluation of memory functions for ion mobility that provide a direct signature of long-ranged ionsolution interactions, $\stackrel{4}{a}$ a signature that we had not anticipated in advance of these simulations.

The theoretical testing naturally relies heavily on molecular simulations that have become accessible in recent years. [Details of the present simulation calculations are provided in the Appendix.] The targets of these calculations were the ion-pair potential-of-meanforce (pmf), the ion-pairing dissociation constant implied by that pmf, then further the mean-square-displacement of the ions individually, their velocity autocorrelation functions, and the corresponding memory functions. To assist the interpretation of these quantities, we also evaluated the dielectric constant of the solvent in the present simulation model.

\section{RESULTS AND DISCUSSION}

The analysis requires that we choose a center for the molecular ions of interest. Radial distribution functions

\footnotetext{
a) Electronic mail: zpeixi@tulane.edu

b) Electronic mail: lpratt@tulane.edu

c)Electronic mail: kyriakos@tulane.edu
}

associated with the chosen centers, (Fig. 1) mid-C for the 1-hexyl-3-methylimidazolium $\left(\mathrm{Hmim}^{+}\right)$ion and $\mathrm{B}$ for the tetrafluoroborate $\left(\mathrm{BF}_{4}{ }^{-}\right)$ion, characterize ion solvation.

The CB-pair pmf, $w(r)$, (Fig. 2) shows strong association. Comparison with a naive dielectric continuum model, $w(r) \approx-q^{2} / 4 \pi \epsilon r$, highlights the molecular structure of the simulation result. The local minimum for $r \approx 0.65 \mathrm{~nm}$ reflects the binding of the $\mathrm{BF}_{4}{ }^{-}$ion to the ring center of the $\mathrm{Hmim}^{+}$ion. Thus multiple binding geometries can be a specific complication in pairing of molecular ions. Additionally, the ionic electric charge is significantly distributed over these molecular structures, so this dielectric model (Fig. 2) is indeed naive for that reason also.

At the longest range here, the computed pmf is foreshortened due to the periodic boundary conditions, i.e., the mean forces between the ions along a cartesian axis will be zero at a boundary face for the simulated result but not for the dielectric model, which is expected to be correct at the longest range. Recognizing that distinction, the dielectric model utilizing the dielectric constant evaluated for the simulation model $n$-pentanol solvent (Fig. 3) over-estimates the maximum binding free energy of this system. This naive dielectric model predicts a more accurate maximum binding free energy when the experimental dielectric constant is utilized, but that comparison has no physical significance.

The dissociation equilibrium ratio is defined by reference to the equilibrium

$$
\mathrm{Hmim} \cdot \mathrm{BF}_{4} \leftrightharpoons \mathrm{Hmim}^{+}+\mathrm{BF}_{4}{ }^{-},
$$

and then

$$
K_{\mathrm{d}}=\frac{\left(\rho_{\mathrm{Hmim}^{+}}\right)\left(\rho_{\mathrm{BF}_{4}-}\right)}{\rho_{\mathrm{Hmim}} \cdot \mathrm{BF}_{4}}
$$

with $\rho_{\alpha}$ the number density of species $\alpha$. The species indicated in Eq. (2) are identified by determining whether the mid-C ... B atom pairs are within a radius $r$ (paired) or not (unpaired). Basic statistical thermodynamics 


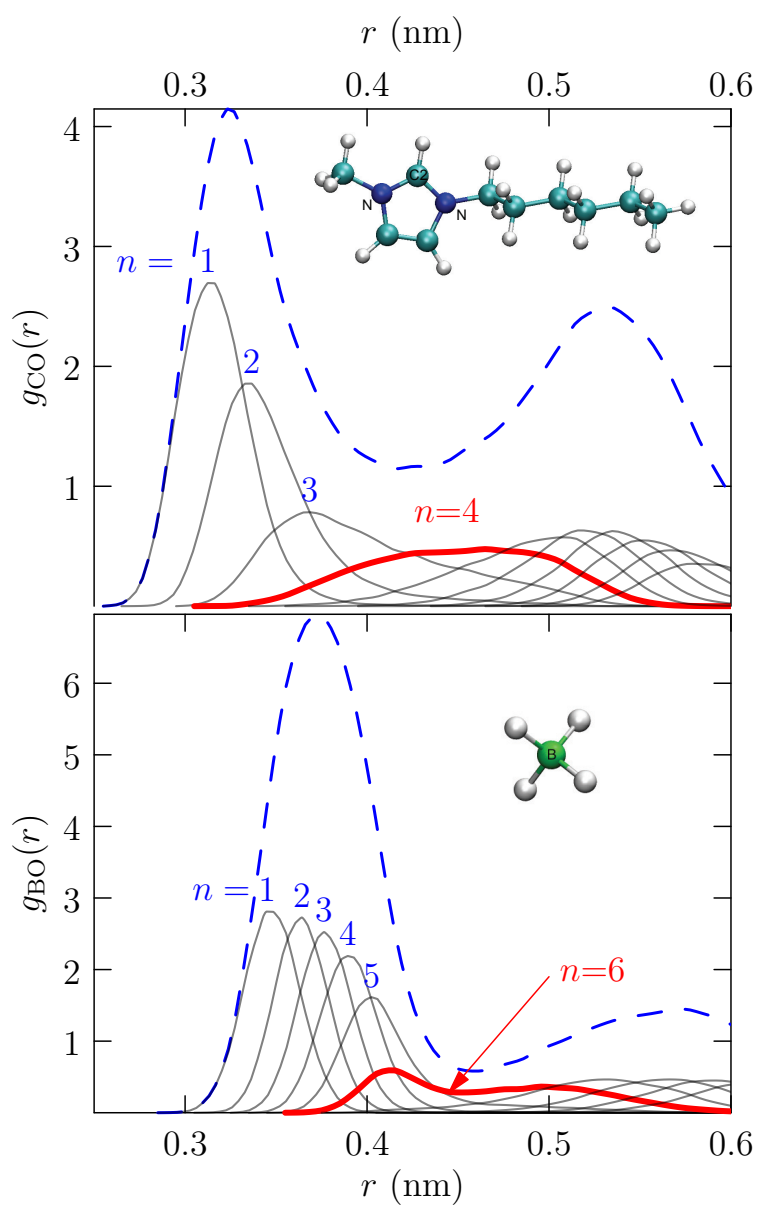

FIG. 1. Radial distribution functions of $n$-pentanol O-atom from the ion centers (upper) middle C-atom of the imidazolium ring and (lower) the B-atom of $\mathrm{BF}_{4}{ }^{-}$, together with the distance-ordered decompositions (grey). For the upper panel $n=1,2,3$ fills out the principal peak and $n=4$ participates in both first and second shell. For the lower panel $n=$ $1, \ldots, 5$ fills out the principal peak and $n=6$ participates in both first and second shell.

identifies this ratid 6 as

$$
\frac{1}{K_{\mathrm{d}}}=\frac{p(n=1)}{p(n=0) \rho_{\mathrm{BF}_{4}-}}
$$

and we acknowledge that $\rho_{\mathrm{BF}_{4}{ }^{-}}=\rho_{\mathrm{Hmim}^{+}} \cdot p(n)$ is the probability of observing $n$ mid-C atoms of $\mathrm{Hmim}^{+}$ions within $r$ of the B-atom of a distinguished $\mathrm{BF}_{4}{ }^{-}$ion. For the infinite dilution circumstances of the present study, the ratio of probabilities (Eq. (3) ) is given precisely by the Poisson distribution formula

$$
\frac{p(n=1)}{p(n=0) \rho_{\mathrm{BF}_{4^{-}}}}=4 \pi \int_{0}^{r} \exp \left[-w\left(r^{\prime}\right) / k_{\mathrm{B}} T\right] r^{\prime 2} \mathrm{~d} r^{\prime} .
$$

If the lengths on the right of Eq. (4) are in $\mathrm{nm}$, and if $K_{\mathrm{d}}$ is $\mathrm{mol} / \mathrm{dm}^{3}$, the factor for conversion of units is

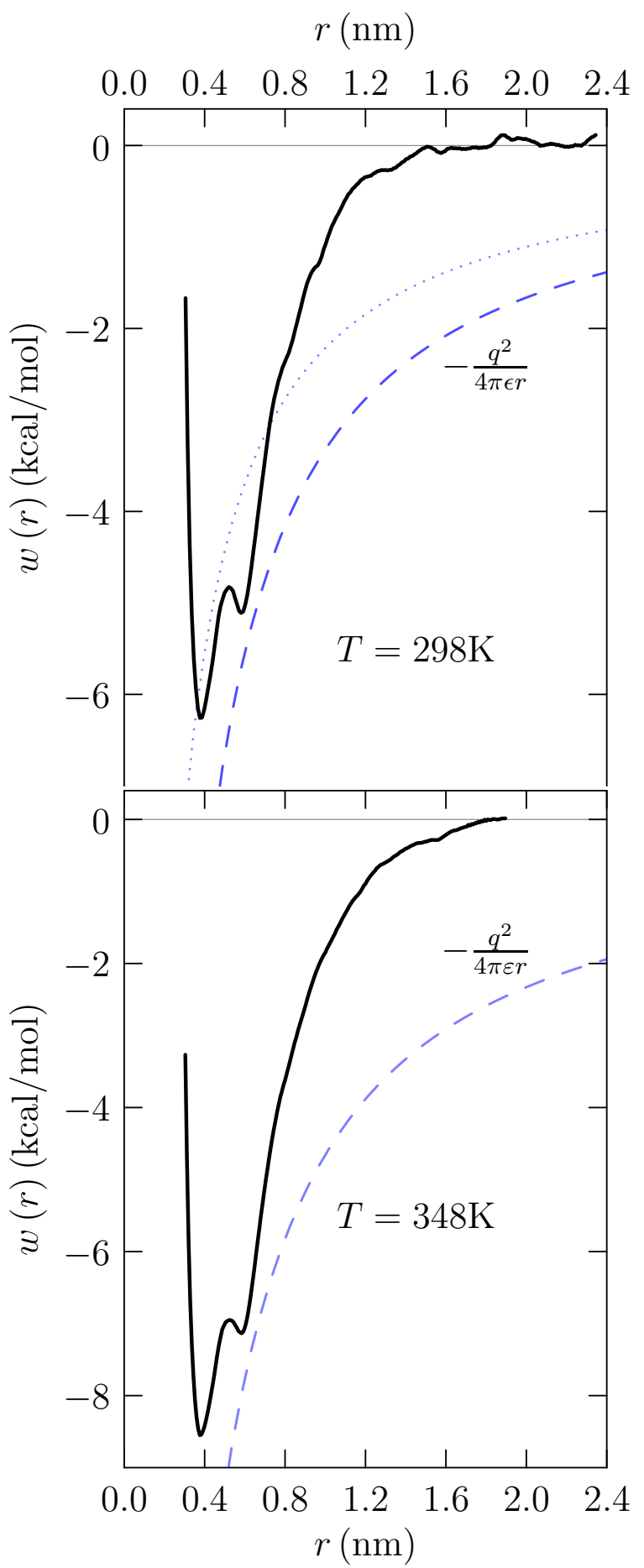

FIG. 2. The CB (Fig. 1) pmf in $n$-pentanol as a function of the CB radial displacement. For the modelled $n$-pentanol solvent, the static dielectric constants are $\epsilon / \epsilon_{0}=7.1$ and 10.0 at $p=1 \mathrm{~atm}$ and $T=298.15 \mathrm{~K}$ and $348.15 \mathrm{~K}$, respectively. The dotted curve utilizes the experimental dielectric constant $(15) !$ 


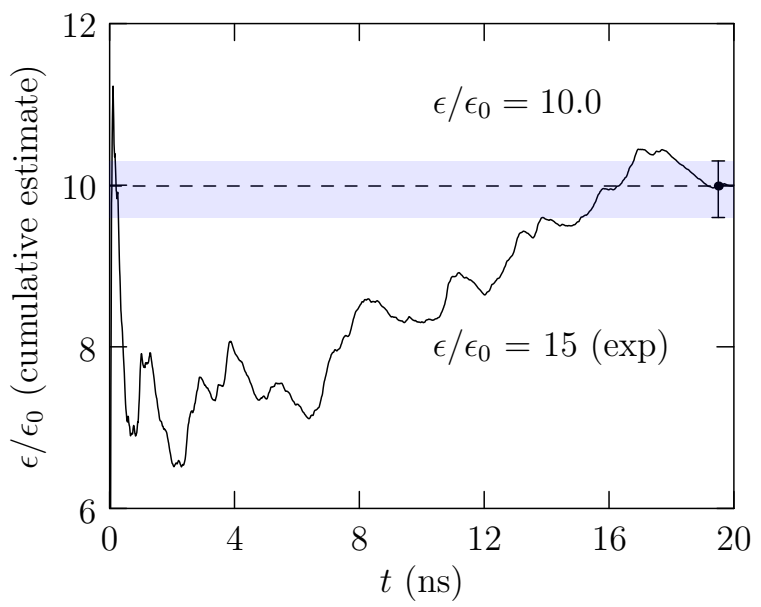

FIG. 3. Computed dielectric constant for the simulated $n$ pentanol liquid at $T=298.15 \mathrm{~K}$ and $p=1 \mathrm{~atm}$. The shaded band indicates the $95 \%$ confidence interval [9.6,10.3], established by bootstrap resampling. See Sec. VB.

$$
\begin{aligned}
& \left.6.023 \times 10^{23} / 10^{24}=0.6023 \mathrm{dm}^{3} /(\mathrm{mol} \mathrm{nm})^{3}\right), \text { so } \\
& K_{\mathrm{d}}=\frac{1}{(0.6023) \times 4 \pi \int_{0}^{r} \exp \left[-w\left(r^{\prime}\right) / k_{\mathrm{B}} T\right] r^{\prime 2} \mathrm{~d} r^{\prime}} .
\end{aligned}
$$

The predicted dissociation constant (Fig. 4) operationally plateaus for $r>0.65 \mathrm{~nm}$, and closely agrees with the experimental result. The ripple near $r \approx 0.6 \mathrm{~nm}$ reflects the second (outer or ring binding) geometry identified with Fig. 2, and thus that second binding mode affects the predicted $K_{\mathrm{d}}$. Overall, we conclude that the present molecular model and simulation predicts correct behavior and magnitudes for the pmf at molecular distances, even though that pmf is foreshortened at longrange as noted above.

The kinetics associated with the mobilities of these ions were characterized first on the basis of the observed mean-square-displacements (Fig. 5)

$$
\frac{\mathrm{d}\left\langle\Delta r(t)^{2}\right\rangle}{\mathrm{d} t}=2 \int_{0}^{t}\langle\vec{v}(0) \cdot \vec{v}(\tau)\rangle \mathrm{d} \tau,
$$

with the underlying velocity autocorrelation functions (Fig. 6)

$$
C(t)=\langle\vec{v}(0) \cdot \vec{v}(t)\rangle /\left\langle|v|^{2}\right\rangle
$$

The indicated velocities are those of the center-of-mass of the extended molecular ions. We also considered a memory function $\gamma(t)$ defined by 10

$$
m \frac{\mathrm{d} C(t)}{\mathrm{d} t}=-\int_{0}^{t} \gamma(t-\tau) C(\tau) \mathrm{d} \tau,
$$

with $m$ the mass of the ion. $\gamma(t)$ provides the autocorrelation of the random forces on the ions. We emphasize the connection to the forces with the notation $\Omega^{2}=\left\langle F^{2}\right\rangle / 3 m k_{\mathrm{B}} T$ so that

$$
\gamma(0)=m \Omega^{2}
$$

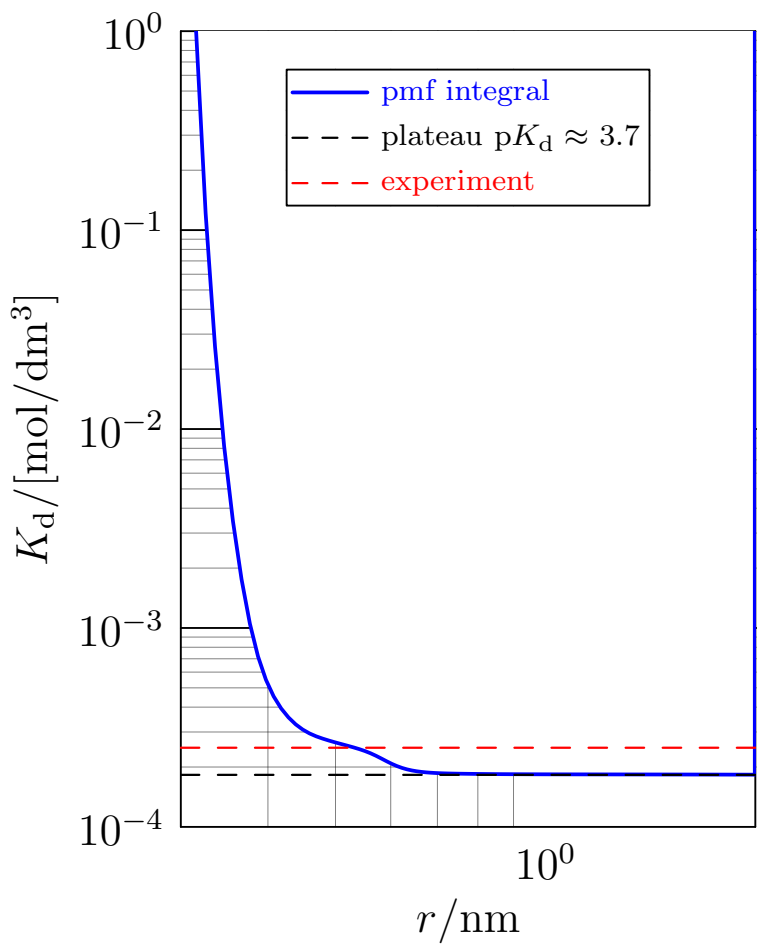

FIG. 4. Evaluation of the dissociation constant $K_{\mathrm{d}}$ of Eq. (4) and comparison with experiment. ${ }^{3}$ The defining integral operationally plateaus for $r>0.65 \mathrm{~nm}$, and closely agrees with the experimental result. The ripple near $r \approx 0.6 \mathrm{~nm}$ reflects the second (outer or ring binding) geometry identified with Fig. 2 . and thus that second binding mode affects the predicted $K_{\mathrm{d}}$.

We extracted $\gamma(t)$ from $C(t)$ on the basis of the relation 10

$$
\int_{0}^{\infty} \mathrm{e}^{-s t} \gamma(t) d t \equiv \tilde{\gamma}(s)=m\left(\frac{1}{\tilde{C}(s)}-s\right)
$$

which follows from Eq. (8), with $\tilde{C}(s)$ the Laplace transform of $C(t)$.

We utilized the Stehfest algorithm 11 to invert the Laplace transform numerically. $\gamma(t)$ is found (Fig. 7) to be non-negative in these cases. Furthermore, $\gamma(t)$ displays two different time regimes, and specifically a slow, exponential time-decay for the longest times analyzed here. The long-time decay recalls the discussion of Wolynes many years agd ${ }^{4}$ of the effects of dielectric friction on ion mobilities. This behavior seems not to be have been observed until now, but Annapureddy and Dang have obtained the analogous result for the autocorrelation of the forces on stationary alkali metal ions in water! 12

\section{CONCLUSION}

The present molecular model and simulation accurately predicts the dissociation constant $K_{\mathrm{d}}$ of 1-hexyl- 


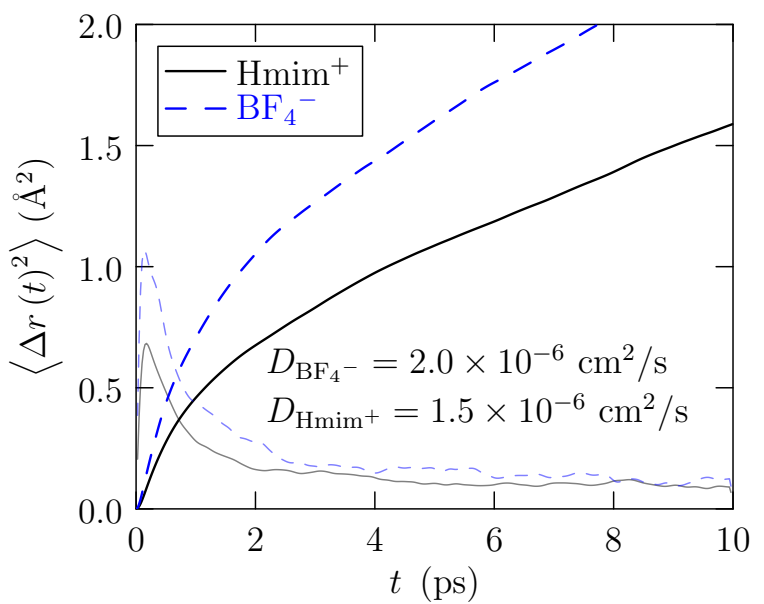

FIG. 5. Mean-square-displacements of the center-of-mass of these molecular ions observed in simulations of the individual ions in $n$-pentanol. The fainter peaked curves in the background are the time-derivatives of the mean-squaredisplacements obtained from Eq. (6). The experimental value $^{[3}$ for the average diffusivity is $1.87 \times 10^{-6} \mathrm{~cm}^{2} / \mathrm{s}$.

3-methylimidazolium and tetrafluoroborate ions in $n$ pentanol in comparison to experiment, and thus the behavior and magnitudes for the ion-pair pmf at molecular distances, even though the dielectric constant of the simulated solvent differs from the experimental value by about $30 \%$. A naive dielectric model does not capture molecule structural effects such as multiple conformations and binding geometries of the $\mathrm{Hmim}^{+}$and $\mathrm{BF}_{4}{ }^{-}$ ion-pairs. Mobilities identify multiple time-scale effects in the autocorrelation of the random forces on the ions, and specifically the slow, exponential time-decay of those long-ranged forces associated here with dielectric friction effects.

\section{ACKNOWLEDGEMENTS}

This work was supported by the National Science Foundation under the NSF EPSCoR Cooperative Agreement No. EPS-1003897, with additional support from the Louisiana Board of Regents. Support from the Louisiana Board of Regents Industrial Ties Research Subprogram (ITRS) and Chevron Energy Technology Company is gratefully acknowledged.

\section{APPENDIX: METHODS}

Potential parameters of ionic liquid (IL) 1-hexyl-3methylimidazolium tetrafluoroborate were taken from de Andrade, $\frac{13}{13}$ which is the AMBER force field with slight modification. $n$-Pentanol force field parameters were taken from AMBER ${ }^{14}$ Partial atom charges of $\mathrm{Hmim}^{+}$ were derived following de Andrade's procedure, while

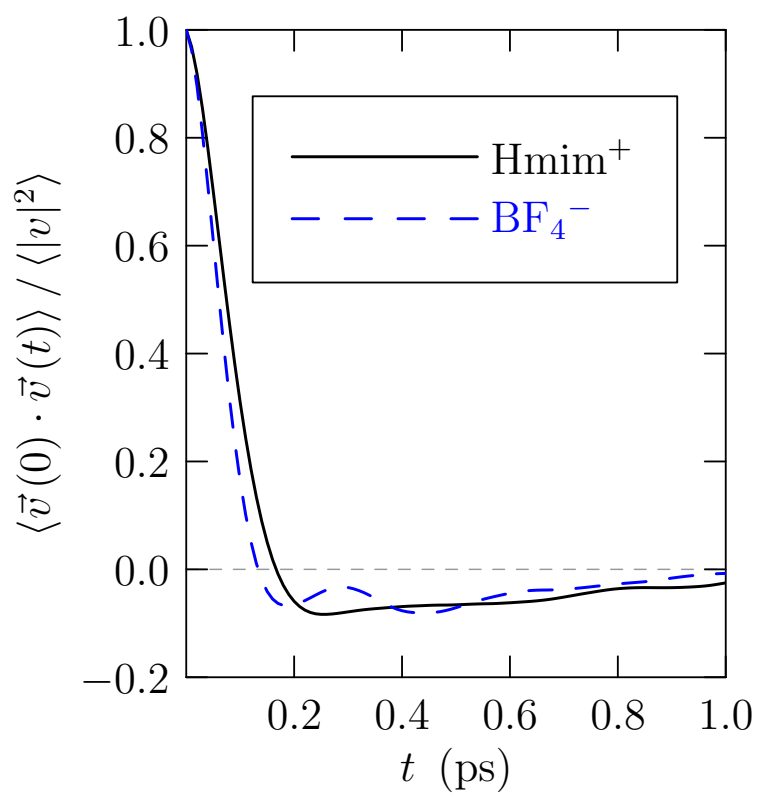

FIG. 6. Autocorrelation functions of the center-of-mass velocities of these molecular ions. The time interval shown here is not sufficient to obtain the self-diffusion coefficients of Fig. 5 On this time interval these functions are qualitatively similar even though the molecular structures of these ions are qualitatively different.

those of $\mathrm{BF}_{4}{ }^{-}$were taken directly from their work $!^{13}$ Partial atom charges used in this work for $n$-pentanol were those developed by Kuhn. $\frac{15}{}$

Our systems were simulated using the AMBER 10 package in an isothermal-isobaric ensemble (NPT) with periodic boundary conditions. The cutoff for nonbonded interactions was $1.7 \mathrm{~nm}$, and Ewald summation was used to calculate electrostatic interactions. The temperature was regulated with Langevin dynamics, while pressure was controlled by Berendsen's weak coupling algorithm. All C-H bonds were constrained by SHAKE algorithm. 16 For the simulation of IL pair in $n$-pentanol, an ion pair was first equilibrated without cutoff in vacuum for $10 \mathrm{~ns}$ to get an optimized geometry. Then it was placed in the center of a $(4.8 \mathrm{~nm})^{3}$ cubic box, which was packed uniformly with $620 n$-pentanol molecules using Packmol 17 For the single ion $/ n$-pentanol systems, $\mathrm{Hmim}^{+}$or $\mathrm{BF}_{4}{ }^{-}$ was simply wrapped up with $620 n$-pentanol molecules in a cubic box of the same size. Aging was 2 ns at $298.15 \mathrm{~K}$ under $1 \mathrm{~atm}$ with $1 \mathrm{fs}$ integration time step. Then $8 \mathrm{~ns}$ production equilibrium run was performed. For the single ion $/ n$-pentanol systems, the production runs extended to $10 \mathrm{~ns}$. Configurations were saved every $1 \mathrm{ps}$ for further analysis. For the velocity autocorrelation function and the mean square displacement of each ion, 1 ns trajectories, with a time step of $2 \mathrm{fs}$, were obtained, saving the phase point at each $10 \mathrm{fs}$. 


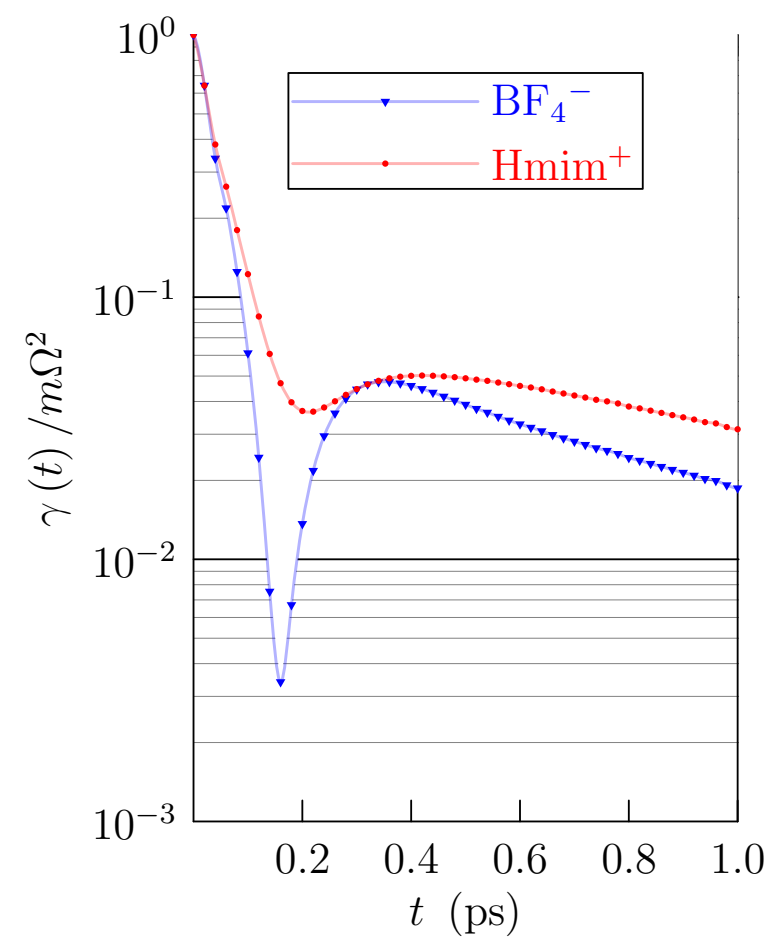

FIG. 7. Logarithm of the normalized autocorrelation function of the random forces on these ions, obtained with the Stehfest algorithm ${ }^{11}$ from the velocity autocorrelation functions (Eq. (6)). That $\gamma(t)$ is non-negative here is obvious but special. $\gamma(t)$ decays approximately exponentially for the largest times shown. This long-time behavior was suggested many years ago in considering dielectric friction on ion mobilities. This behavior seems not to be have been observed until now, but Annapureddy and Dang have also obtained the analogous result for the autocorrelation of the forces on stationary alkali metal ions in water. 12

\section{A. The pmf and WHAM calculations}

To obtain the ion-pair pmf, a reaction coordinate was defined as the distance between the central carbon of the imidazolium ring (Fig. 1) and boron atom of the $\mathrm{BF}_{4}{ }^{-}$ion. Window calculations then utilized a harmonic stratifying potential with a force constant of $100 \mathrm{kcal} /(\mathrm{mol} \cdot \mathrm{nm})$ for windows from $0.3 \mathrm{~nm}$ to $2.35 \mathrm{~nm}$ (at $298.15 \mathrm{~K}$ ), and from $0.3 \mathrm{~nm}$ to $1.95 \mathrm{~nm}$ (at $348.15 \mathrm{~K}$ ), with an increment of $0.05 \mathrm{~nm}$. Typically, the system was equilibrated for $1 \mathrm{~ns}$ to initiate each window simulation, and then followed by 10 ns production run. For window separations greater than $1.7 \mathrm{~nm}$, the initial configuration for the next window was taken from the last configuration from the previous MD simulation. The weighted histogram analysis method (WHAM) ${ }^{18}$ was used to synthesize the final pmf profile. For the calculation at $348.15 \mathrm{~K}$, the system consisted of an IL pair and $340 n$-pentanol molecules in a $(4 \mathrm{~nm})^{3}$ cubic cell.

\section{B. $n$-Pentanol Dielectric Constant}

Assessment of the static dielectric constant of liquid $n$-pentanol is required in considering the molecular pair potential of the mean forces for $\mathrm{Hmim}^{+} \ldots \mathrm{BF}_{4}{ }^{-}$in $n$ pentanol. The dielectric constant for this model was obtained by standard simulation methods $\frac{1920}{20}$ The calculation treated $620 n$-pentanol molecules under standard periodic boundary conditions. The results $\left(\epsilon / \epsilon_{0}=7.1\right.$ and 10.0) were extracted from averaging over $20 \mathrm{~ns}$ of simulation trajectory under constant pressure conditions, and at constant temperatures of $298.15 \mathrm{~K}$ and $348.15 \mathrm{~K}$, respectively. These computed values for the dielectric constant of the modelled solvent are about $30 \%$ smaller than experimental values (Fig. 3). 
${ }^{1}$ T. Welton, Chem. Rev. 99, 2071 (1999).

${ }^{2}$ W. Kunz, ed., Specific Ion Effects (World Scientific, 2010).

${ }^{3}$ P. Zhu, T. V. Harris, M. S. Driver, C. B. Campbell, L. R. Pratt, and K. D. Papadopoulos, J. Phys. Chem. C 113, 16458 (2009). ${ }^{4}$ P. G. Wolynes, J. Chem. Phys. 68, 473 (1978).

${ }^{5}$ CRC Handbook of Chemistry and Physics, 92nd ed. (CRC Press, 2011-2012) Chap. 6, pp. 6-187, internet version.

${ }^{6}$ T. L. Beck, M. E. Paulaitis, and L. R. Pratt, The Potential Distribution Theorem and Models of Molecular Solutions (Cambridge University Press, 2006).

${ }^{7}$ S. Chempath, L. R. Pratt, and M. E. Paulaitis, J. Chem. Phys. 130, 054113(1 (2009).

${ }^{8}$ D. Asthagiri, P. D. Dixit, S. Merchant, M. E. Paulaitis, L. R. Pratt, S. B. Rempe, and S. Varma, Chem. Phys. Letts. 485, 1 (2010).

${ }^{9}$ P. Zhu, Y. You, L. R. Pratt, and K. D. Papadopoulos, J. Chem. Phys. 134, 054502 (2011).

${ }^{10}$ R. Zwanzig, Nonequilibrium Statistical Mechanics (Oxford, 2001).
${ }^{11}$ H. Stehfest, Comm. ACM 13, 47 (1970).

${ }^{12}$ H. V. R. Annapureddy and L. X. Dang, "Specific ion interactions between carboxylate anions and alkali metal cations," Tech. Rep. (2012).

${ }^{13}$ J. de Andrade, E. S. Böes, and H. Stassen, J. Phys. Chem. B 106, 13344 (2002).

${ }^{14}$ V. Hornak, R. Abel, A. Okur, B. Strockbine, A. Roitberg, and C. Simmerling, Proteins 65, 712 (2006).

${ }^{15}$ H. Kuhn, J. Coll. Interfac. Sci. 249, 152 (2002).

${ }^{16}$ J. Ryckaert, G. Ciccotti, and H. Berendsen, J. Comp. Phys. 23, 327 (1977).

${ }^{17}$ L. Martínez, R. Andrade, E. G. Birgin, and J. M. Martínez, J. Comp. Chem. 30, 2157 (2009).

${ }^{18} \mathrm{~A}$. Grossfield, "WHAM: the weighted histogram analysis method, version 2.0.4," http://membrane.urmc.rochester.edu/content/wham.

${ }^{19}$ Y. Wu, H. L. Tepper, and G. A. Voth, J. Chem. Phys. 124, 024503 (2006).

${ }^{20}$ L. Yang, B. H. Fishbine, A. Migliori, and L. R. Pratt, J. Chem. Phys. 132, 044701(1 (2010). 